SLAC-PUB-10022

OSU-RN-294

\title{
Theorems on Estimating Perturbative Coefficients in Quantum Field Theory and Statistical Physics
}

\author{
Mark A. Samuel and Stephen D. Druger
}

Submitted to International Journal of Theoretical Physics 


\begin{abstract}
We present rigorous proofs for several theorems on using Padé approximants to estimate coefficients in Perturbative Quantum Field Theory and Statistical Physics. As a result, we find new trigonometric and other identities where the estimates based on this approach are exact. We discuss hypergeometric functions, as well as series from both Perturbative Quantum Field Theory and Statistical Physics.
\end{abstract}




\section{INTRODUCTION}

Recently, we proposed ${ }^{1.5}$ a method of estimating coefficients in Perturbative Quantum Field Theory and Statistical Physics with error bars for each estimate. The method makes use of Padé approximants and yields a Padé approximant approximation (PAP). There are many good references for Padé approximants, such as for example Refs. 6-10. We begin by defining the Padé approximant

$$
[N / M]=\frac{a_{0}+a_{1} x+a_{2} x^{2}+\ldots+a_{N} x^{N}}{1+b_{1} x+b_{2} x^{2}+b_{3} x^{3}+\ldots+b_{M} x^{M}}
$$

to the series

$$
S=S_{0}+S_{1} x+\ldots+S_{N+M} x^{N+M}
$$

where we set

$$
[N / M]=S+O\left(x^{N+M+1}\right)
$$


We have written a computer program that solves Eq. (1.3) numerically and then predicts the coefficient of the next term $S_{N+M+1}$. It works for arbitrary $N$ and $M$. Furthermore, we have derived algebraic formulae for the $[N / 1],[N / 2],[N / 3],[N / 4]$, [N/5], and [N/6] PAP's, where $N$ is arbitrary.

To illustrate the method, consider the simple example

$$
\frac{\ln (1+x)}{x}=1-\frac{x}{2}+\frac{x^{2}}{3}-\frac{x^{3}}{C}
$$

We write the $[1 / 1]$ Padé as

$$
[1 / 1]=\frac{a_{0}+a_{1} x}{1+b_{1} x}
$$

It is easy to show that $a_{0}=1, b_{1}=2 / 3, a_{1}=1 / 6$, and $C=9 / 2$. We can see that the prediction for $C$ is close to the correct value $C=4$. For $x=1$, we get $[1 / 1]=7 / 10$, close to the correct result, $\ln 2=0.6931$. This is much better than the partial sum

$$
1-\frac{1}{2}+\frac{1}{3}=\frac{5}{6}=0.8333
$$

By going to higher order, it is easy to show that 


$$
[1 / 2]=\frac{1+\frac{x}{2}}{1+x+\frac{x^{2}}{6}}
$$

and for $x=1$ we obtain

$$
m[1 / 2]=\frac{9}{13}=0.6923
$$

very close to $\ln 2$. The PAP is $7 / 36=0.1944$ very close to the correct value of $1 / 5$.

As discussed in our recent article, the error bars are obtained by taking the reciprocals

$$
r_{n}=\frac{1}{S_{n}}
$$

and finding the PAP for $r_{n+1}$, and then taking the reciprocal. Then we consider the differences

$$
t_{n}=r_{n+1}-r_{n}
$$


and find the PAP for $t_{n}$. We then have

$$
r_{n+1}=r_{n}+t_{n}
$$

and then take the reciprocal

$$
S_{n+1}=\frac{1}{r_{n+1}} .
$$

Our error bar is calculated from the difference between the results from Eqs. (1.9) and (1.12).

\section{THEOREMS}

We consider the general series

$$
S=\sum_{n} S_{n} x^{n}
$$

A. Sums of geometric series

If $S_{n}$ is a sum of $M$ geometric series then the [N/M] PAP for $N \geq M-1$ is exact. Proof: For $M=2$,

$$
S_{n}=a r^{n}+b s^{n}
$$

and 


$$
S=\sum_{n=0}^{\infty} S_{n} x^{n}=\frac{a}{1-r x}+\frac{b}{1-s x}
$$

so that

$$
S=\frac{(a+b)-(a s+b r) x}{(1-r x)(1-s x)}
$$

and the [1/2] and higher PAP's are exact.

To prove the general case we use mathematical induction. Assume that the theorem is true for the [M-1/M] PAP. Now for $M \rightarrow M+1$, we have

$$
\frac{P_{M-1}}{Q_{M}}+\frac{g}{1-a x}=\frac{P_{M-1}+g Q_{M}}{Q_{M}(1-a x)}
$$

and the $[M / M+1]$ PAP is exact. Here

$$
S_{n}=a r^{n}+b s^{n}+\ldots+g w^{n}
$$

and $P_{M}$ and $Q_{M}$ are polynomials of degree $M$.

B. Signs of geometric series

For

$$
S_{n}=(-1)^{n} C_{m} a r^{n}
$$


the $[\mathrm{m}-1 / \mathrm{m}] \mathrm{PAP}$ is exact.

Proof: The proof is easily obtained by recognizing that the series in Eq. (2.7) is just a sum of geometric series and by next using Theorem A.

C. A sufficient condition for PAP's to be accurate

If we define

$$
g(n)=\frac{d^{2} \ln S_{n}}{d n^{2}}
$$

then a sufficient (but not necessary) condition for the PAP's to be accurate is

$$
\lim _{n \rightarrow \infty} g(n)=0 \text {. }
$$

Proof: We define

$$
A_{n} \equiv 1+\varepsilon_{n} \equiv \frac{S_{n} S_{n+2}}{\left(S_{n+1}\right)^{2}}
$$

and hence 


$$
A_{n}=1+\varepsilon_{n}=e^{g(n)}
$$

The \% error is expressible in terms of the $\varepsilon_{\mathrm{n}}$ and if $g(n) \rightarrow 0$ then $\varepsilon_{\mathrm{n}} \rightarrow 0$ and the PAP is accurate.

D. A generalization of Theorem $\mathrm{C}$

If, in addition to the conditions of Theorem $C$ for $S_{n}$ we generalize to a series

$$
T=\sum_{n} T_{n} x^{n}
$$

where

$$
T_{n}=(-1)^{\cdot{ }^{*}} S_{n}
$$

then the $[\mathrm{m}-1 / \mathrm{m}]$ and higher PAP's will be accurate.

Proof:

$$
A_{n}^{\prime}=1+\varepsilon_{n}^{\prime}=(-1)^{n}{ }^{n} C_{m-2} A_{n}
$$

where $A_{n}$ is given by Eq. (24). Then we use Theorems $B$ and $C$ to prove Theorem $D$. For further details, see Ref. 2. 
E. Polynomials of the $\mathrm{n}^{\text {th }}$ degree

For $S_{n}=P_{n}$ where $P_{n}$ is a polynomial of degree $n$, the [N/M] PAP's are exact, where $\mathrm{M}=\mathrm{n}+1$ and $\mathrm{N} \geq \mathrm{M}-1$.

Proof: By differentiating

$$
S=\sum_{n} x^{n}=(1-x)^{-1}
$$

and multiplying by $\mathrm{x}$, we obtain

$$
\sum_{n}(a n+b) x^{n}=\frac{(a-b) x+b}{(1-x)^{2}}
$$

and the $[N / 2]$ is exact for $N \geq 1$. Now by induction we can easily obtain the desired result.

It should be emphasized that in all cases once the [N/M] PAP's are exact for $N \geq M-1$, the results remain exact for all higher order PAP's $\left[N^{\prime} / M^{\prime}\right]$ for $M^{\prime}>M$ and $\mathrm{N}^{\prime}>\mathrm{M}^{\prime}-1$.

\section{SOME NEW TRIGONOMETRIC IDENTITIES}

If we consider the series given by

$$
S_{n}=\sin [(n+1) \theta+\delta]
$$


where $\theta$ and $\delta$ are arbitrary we will prove that the [N/M] PAP's are exact for $M \geq 2$ and $\mathrm{N} \geq \mathrm{M}-1$. This leads to new trigonometric identities corresponding to each of the [N/2], [N/3], [N/4], etc., PAP's.

From Eq. (3.1) it can easily be shown that

$$
\begin{gathered}
S=\sum_{n=0}^{\infty} S_{n} x^{n} \\
=\frac{x \cos (\theta+\delta) \sin \theta+\sin (\theta+\delta)(1-x \cos \theta)}{1-2 x \cos \theta+x^{2}} .
\end{gathered}
$$

Hence the $[N / M]$ PAP's are exact for $M \geq 2, N \geq M-1$. With $\delta=0$, Eq. (3.2) becomes

$$
S=\frac{\sin \theta}{1+x^{2}-2 x \cos \theta}
$$

and, hence, the $[0 / 2] \mathrm{PAP}$ is exact.

Similarly for

$$
S_{n}=\cos [(n+1) \theta+\delta]
$$

we can obtain

$$
S=\frac{\cos (\theta+\delta)(1-x \cos \theta)-x \sin (\theta+\delta) \sin \theta}{1-2 x \cos \theta+x^{2}}
$$

In this case, however, if we set $\delta=0$, we obtain 


$$
S=\frac{\cos \theta-x}{1-2 x \cos \theta+x^{2}},
$$

and the [0/2] PAP is not exact!

Now, for each [N/M] PAP that is exact we will find a trigonometric identity.

We begin with $\mathrm{M}=2$. The:[1/2] $\mathrm{PAP}$ is given by $S_{4}=N / D$ where

$$
N=2 S_{1} S_{2} S_{3}-S_{0} S_{3}^{2}-S_{2}^{3}
$$

and

$$
D=S_{1}^{2}-S_{0} S_{2}
$$

with the $S_{n}$ given by Eq. (3.1). Thus we have the identity

$$
N-S_{4} D \equiv 0
$$

which becomes

$$
\begin{gathered}
2 \sin (2 \theta+\delta) \sin (3 \theta+\delta) \sin (4 \theta+\delta) \\
-\sin (\theta+\delta) \sin ^{2}(4 \theta+\delta)-\sin ^{3}(3 \theta+\delta) \\
-\sin (5 \theta+\delta)\left[\sin ^{2}(2 \theta+\delta)-\sin (\theta+\delta) \sin (3 \theta+\delta)\right]=0 .
\end{gathered}
$$


Now one can step up in $n, S_{n} \rightarrow S_{n+1}$, and obtain another identity. This procedure can be repeated indefinitely. One can also step down in $n, S_{n} \rightarrow S_{n-1}$, where we set $S_{-1}=0$. This gives a simpler identity, which can be obtained from simple known identities, for the [0/2] PAP. But we must set $\delta=k \pi, k=0,1,2, \ldots$, yielding

$$
2 \sin \theta \sin (2 \theta) \sin (3 \theta)-\sin ^{3}(2 \theta)-\sin (4 \theta) \sin ^{2} \theta=0 .
$$

One can also use Eq. (31) to obtain the same identities for $\cos [(n+1) \theta+\delta]$. However, in this case there is no [0/2] identity for $\delta=0$.

We now turn to $M=3$. The [2/3] PAP is given by

$$
S_{6}=A / B
$$

where

$$
\begin{gathered}
A=2 S_{2}^{2} S_{3} S_{5}-2 S_{1} S_{3}{ }^{2} S_{5}+2 S_{0} S_{3} S_{4} S_{5} \\
-2 S_{1} S_{2} S_{4} S_{5}+S_{1}^{2} S_{5}^{2}-S_{0} S_{2} S_{5}{ }^{2}+S_{2}{ }^{2} S_{4}{ }^{2} \\
-3 S_{2} S_{3}^{2} S_{4}+2 S_{1} S_{3} S_{4}{ }^{2}-S_{0} S_{4}{ }^{3}+S_{3}{ }^{4}
\end{gathered}
$$

and

$$
B=S_{2}^{3}-2 S_{1} S_{2} S_{3}+S_{0} S_{3}^{2}-S_{0} S_{2} S_{4}+S_{1}^{2} S_{4}
$$

Again we use Eqs. (3.1) and (3.4), but this time there are two identities in each case 


$$
A=0 \text { and } B=0 \text {. }
$$

The first part of Eq. (3.15) yields new identities, but the second part gives only previous identities for $M=2$. This is in accordance with a theorem presented in an earlier paper. ${ }^{2}$ We can again step up in $n, S_{n} \rightarrow S_{n+1}$, and obtain more identities for both $\sin [(n+1) \theta+\delta]$ and $\cos [(n+1) \theta+\delta]$. This process may be repeated as many times as desired. Now we may very easily also step down in $n, S_{n} \rightarrow S_{n-1}$, where we set $S_{-1}=0$. This gives identities for the [1/3]. In this case the identity obtained results from

$$
A-B S_{6}=0
$$

for both sine and cosine, with $\delta \neq n \pi$. For the sine case, if $\delta=k \pi$, we obtain

$$
A=0 \text { and } B=0 \text {. }
$$

If we step down once more to the [0/3] PAP and set $\delta=k \pi$, then we obtain an identity for sine, but not for cosine.

Although this process can be continued indefinitely for $M=4,5,6, \ldots$, the formulae become increasingly complicated, as will soon be seen. So we present results for only one more value of $M$, namely $M=4$. The [3/4] PAP is given by 


$$
S_{8}=C / D
$$

where

$$
\begin{gathered}
C=2\left(2 S_{2} S_{3} S_{4}^{2} S_{7}-S_{3}^{3} S_{4} S_{7}-S_{1} S_{4}^{3} S_{7}\right. \\
-S_{2}^{2} S_{4} S_{5} S_{7}+S_{1} S_{3} S_{4} S_{5} S_{7}-S_{3}^{2} S_{4}^{2} S_{6}+2 S_{1} S_{4}^{2} S_{5} S_{6} \\
+S_{2}^{2} S_{4} S_{6}^{2}-S_{1} S_{3} S_{4} S_{6}^{2}-S_{2} S_{3} S_{4} S_{5} S_{6}-S_{2} S_{4}^{3} S_{6} \\
+S_{3}^{2} S_{4} S_{5}^{2}+S_{2} S_{4}^{2} S_{5}^{2}-2 S_{3} S_{4}^{3} S_{5}-S_{1} S_{4} S_{5}^{3}+S_{2} S_{3}^{2} S_{5} S_{7} \\
+S_{0} S_{4}^{2} S_{5} S_{7}+S_{1} S_{2} S_{5}^{2} S_{7}-S_{0} S_{3} S_{5}^{2} S_{7}-S_{2}^{2} S_{4} S_{5} S_{7} \\
-S_{1} S_{3} S_{4} S_{5} S_{7}-S_{2}^{2} S_{3} S_{6} S_{7}-S_{0} S_{3} S_{4} S_{6} S_{7}-S_{1}^{2} S_{5} S_{6} S_{7} \\
+S_{0} S_{2} S_{5} S_{6} S_{7}+S_{1} S_{2} S_{4} S_{6} S_{7}^{2}+S_{1} S_{3}^{2} S_{6} S_{7}-S_{1} S_{2} S_{3} S_{7} \\
-S_{3}^{3} S_{5} S_{6}-S_{1} S_{3} S_{4} S_{6}^{2}-S_{1} S_{2} S_{5} S_{6}^{2}+S_{1} S_{3} S_{5}^{2} S_{6} \\
\left.+S_{0} S_{3} S_{5} S_{6}^{2}-S_{2} S_{3} S_{5}^{3}\right)-3 S_{0} S_{4} S_{5}^{2} S_{6}+S_{2}^{3} S_{7}^{2}+S_{0} S_{3}^{2} S_{7}^{2} \\
+S_{1}^{2} S_{4} S_{7}^{2}-S_{0} S_{2} S_{4} S_{7}^{2}+S_{2} S_{3}^{2} S_{6}^{2}+S_{1}^{2} S_{6}^{3}-S_{0} S_{2} S_{6}^{3} \\
+S_{3}^{2} S_{4} S_{5}^{2}+S_{2}^{2} S_{5}^{2} S_{6}+S_{4}^{5}+S_{2} S_{4}^{2} S_{5}^{2}+S_{3}^{2} S_{4}^{2} S_{6} \\
\quad-S_{2} S_{4}^{3} S_{6}+S_{0} S_{4}^{2} S_{6}^{2}+S_{0} S_{5}^{4}
\end{gathered}
$$

and 


$$
\begin{gathered}
D=2\left(S_{1} S_{3}^{2} S_{5}+S_{1} S_{2} S_{4} S_{5}-S_{1} S_{3} S_{4}^{2}\right. \\
\left.-S_{2}^{2} S_{3} S_{5}-S_{1} S_{2} S_{3} S_{6}-S_{0} S_{3} S_{4} S_{5}\right)+3 S_{2} S_{3}^{2} S_{4} \\
-S_{3}^{4}-S_{1}^{2} S_{5}^{2}+S_{1}^{2} S_{4} S_{6}+S_{0} S_{4}^{3}+S_{0} S_{2} S_{5}^{2} \\
+S_{0} S_{3}^{2} S_{6}-S_{0} S_{2} S_{4} S_{6}+S_{2}^{3} S_{6}-S_{2}^{2} S_{4}^{2}
\end{gathered}
$$

The identities for the [3/4] PAP are

$$
C=0 \text { and } D=0 \text {. }
$$

for arbitrary $\delta$ in both the sine and cosine cases. Again we may step up $S_{n} \rightarrow S_{n+1}$, etc., and obtain new identities. We may also step down to the [2/4] PAP. For the [1/4] PAP the identity is obtained for arbitrary $\delta$ from

$$
C-D S=0
$$

for arbitrary $\delta$. For $\delta=k \pi$ we obtain for the sine case the identities

$$
C=0 \text { and } D=0
$$

For the [0/4], for $\delta=k \pi$, sine works but not cosine.

We believe these identities are new, except for the [0/2] PAP. We would be interested in hearing from anyone who believes any of these identities are already known. 


\section{THE GENERALIZED HYPERGEOMETRIC FUNCTION}

The hypergeometric function ${ }_{k} F_{m}$ represents a large number of elementary functions. Thus we can consider PAP's for many functions at once. We will see that the PAP's are accurate for arbitrary $\mathrm{k}$ and $\mathrm{m}$ and a large number of parameters a, b, c, ... . For many examples of how numerous mathematical functions can be expressed in terms of the hypergeometric function ${ }_{2} F_{1}$ or the confluent hypergeometric function ${ }_{1} F_{1}$ see, for example, the books by Arfken, ${ }^{12}$ by Abramowitz and Stegun, ${ }^{13}$ and by Gradshteyn and Rhyzik. ${ }^{14}$

Consider the hypergeometric series given by

$$
S_{n}=\frac{(a)_{n}(b)_{n}}{(c)_{n} n !}
$$

where

$$
(a)_{n}=a(a+1) \ldots(a+n+1)
$$

and hence

$$
{ }_{2} F_{1}(a, b, c ; x)=\sum_{n=0}^{\infty} S_{n} x^{n}
$$

For the [N/2] PAP the percentage error is given by $100 \mathrm{p}$ where 


$$
p=\frac{\varepsilon_{N}^{2} / \epsilon_{N-1}-\epsilon_{N+1}\left(1+\epsilon_{N}\right)^{2}}{\left(1+\varepsilon_{N}\right)^{2}\left(1+\varepsilon_{N+1}\right)} ; N \geq 1
$$

It can be shown for the ${ }_{2} F_{1}$ hypergeometric function that

$$
p \sim \frac{-2 B(1+B)}{N^{4}}
$$

where

$$
B=c+1-a-b
$$

and, hence, the PAP's quickly become accurate as $N \rightarrow \infty$. For ${ }_{1} F_{1}(a, c ; x)$

$$
p \sim+\frac{2}{N^{2}}
$$

and for ${ }_{2} \mathrm{~F}_{0}(\mathrm{a}, \mathrm{b}, \mathrm{c} ; \mathrm{x})$

$$
p \sim-\frac{2}{N^{2}} .
$$

For the general case ${ }_{k} F_{m}$, if $k \neq m+1$,

$$
p--\frac{2 A}{N^{2}}
$$


where

$$
A=k-(m+1),
$$

and if $\mathrm{k}=\mathrm{m}+1$, then

$$
p-\frac{-2 B(1+B)}{N^{4}}
$$

where

$$
B=2+k^{2}-2 k+m-k m+\sum_{i=1}^{m} c_{i}-\sum_{i=1}^{k} a_{i} \text {. }
$$

In general if

$$
\begin{gathered}
\varepsilon_{n} \sim A / N \\
p \sim-2 A^{2} / N^{2},
\end{gathered}
$$

and if

$$
\begin{gathered}
\varepsilon_{n}-B / N^{2}, \\
p \sim-2 B(1+B) / N^{4} .
\end{gathered}
$$


To check the behavior for $M \neq 2$ we have written a computer program that scans over $a, b, c$ values (skipping over integers) and evaluates the corresponding PAP's. The parameters $a, b$, and c vary from -5.0 to 5.0 in steps of 0.125 . For each [N/M] PAP, the fractional error $p$ is evaluated, and the maximum and minimum values of $p$ listed as TESTMAX and TESTMIN, respectively. The results for ${ }_{2} F_{1},{ }_{1} F_{1}$, and ${ }_{2} F_{0}$ are presented in Tables I, II, and III, respectively. It can be seen that the TESTMIN and TESTMAX values decrease rapidly in going to progressively higher order. We have listed only diagonal PAP's, but other Padé's were also computed and gave very good results.

\section{OTHER EXAMPLES OF EXACT PAP'S}

Other examples can be found in which PAP's are exact. Any series whose sum is a rational fraction of two polynomials

$$
S=\sum_{n=0}^{\infty} S_{n} x^{n}=\frac{P_{N_{0}}(x)}{Q_{M_{0}}(x)}
$$

will be exact for the $[N / M]$ PAP where $N \geq N_{0}$ and $M \geq M_{0}$. Some examples include

$$
S_{n}=(2 n+1) ; \quad N_{0}=1, M_{0}=2
$$




$$
\begin{gathered}
S_{n}=(n+1)^{2} ; \quad N_{0}=1, M_{0}=3 \\
S_{n}=(2 n+1)^{2} ; \quad N_{0}=2, M_{0}=3 \\
S_{n}=(a+n d) ; \quad N_{0}=1, M_{0}=2 \\
S_{n}=(n+1) ; \quad N_{0}=0, M_{0}=2 \\
S_{n+1}-S_{n}=(n+2), S_{0}=1 ; \quad N_{0}=0, M_{0}=3
\end{gathered}
$$

and

$$
S_{n}=1 ; \quad N_{0}=0, M_{0}=1
$$

\section{NON-SINGLET MOMENTS OF DEEP INELASTIC STRUCTURE FUNCTIONS IN QCD}

In this section we make use of some recent results of Larin, van Rittergen, and Vermeseren. ${ }^{15}$ They have calculated the next-to-next leading QCD approximations for non-singlet moments of deep inelastic structure functions, in the 
leading twist approximation, for the moments $\mathrm{N}=2,4,6,8$ of the non-singlet deep inelastic structure function $F_{L}$. They have calculated the three-loop anomalous dimensions of the corresponding non-singlet operators and the three-loop coefficient functions of the structure factor $F_{L}$, in the leading twist and massless quark approximation.

We present our results in Tables IV.XI. In each case, we estimate the $O\left(\alpha_{s}{ }^{3}\right)$ coefficient and compare our estimate with the now-known Larin et al. result. We neglect the term that depends on the sum of the quark charges $\Sigma q_{p}$ since the term is small in all cases of interest. We present our results for $N_{f}=3,4,5$, where $N_{f}$ is the number of quark flavors. We then present our estimates for the next (unknown) $O\left(\alpha^{4}{ }^{4}\right)$ coefficients, in each case.

In Table IV we present results for $C_{L, 2}$. It is seen that, for $N_{f}=3,4,5$, our estimates are within the error-bars, for the $O\left(\alpha_{1}^{3}\right)$ terms and we estimate the next (unknown) $O\left(\alpha_{8}{ }^{4}\right)$ terms. Table $\mathrm{V}$ shows the results for $\mathrm{C}_{\mathrm{L}, 4}$, Table VI for $\mathrm{C}_{\mathrm{L}, 6}$, and Table VII for $\mathrm{C}_{\mathrm{L}, \mathrm{8}}$.

In Tables VIII-XI we present our results for the anomalous dimensions $\gamma_{2}, \gamma_{4}$, $\gamma_{6}$, and $\gamma_{8}$. Here again, in each case, our estimates are within the error bars of the Larin et al. results for $O\left(\alpha_{1}{ }^{3}\right)$ and we estimate the next (unknown) $O\left(\alpha_{s}{ }^{4}\right)$ term. For further details on how we obtain our error bars, see Ref. 11 .

\section{EXAMPLES FROM STATISTICAL PHYSICS}

In this section we consider two examples ${ }^{16}$ from statistical physics. They are 
the low temperature ferromagnetic susceptibility coefficients in the Ising Model. Table XII gives the results for the honey-combed (hc) lattice and the results for the square (sq) lattice are shown in Table XIII.

It can be seen that the results are excellent and that the \% error decreases in going to higher order. In all cases the estimates are within $2 \sigma$ of the exact results for the known coefficients and the next (unknown) coefficient is predicted. For the hc lattice the estimate is $538,596,000 \pm 10,500$ and for the square lattice it is $185,000,000 \pm 55,000,000$.

\section{CONCLUSIONS}

We have proved several theorems on using Padé approximants to estimate coefficients in Perturbative Quantum Field Theory and Statistical Physics. These theorems give sufficient conditions for the PAP method of estimating the next term in a series expansion to work. In addition, we have presented new trigonometric identities which we obtained as a result of the PAP being exact. We have also considered the generalized hypergeometric function, for which the method works. As a result, many series are dealt with at the same time, since the hypergeometric function can represent many elementary functions merely by changing the parameters.

We have considered several series from QCD. These are for the non-singlet moments of deep inelastic structure functions. We have also considered two series from statistical physics. These are the low temperature ferromagnetic susceptibility 
coefficients in the Ising model.

In all cases, the method works beautifully! Thus the information needed for estimating the next term in perturbative series is, in fact, contained in the lowerorder results.

\section{ACKNOWLEDGEMENTS}

One of us (MAS) thanks the theory groups at the Stanford Linear Accelerator Center and at the Argonne National Laboratory for their kind hospitality. He also thanks the following people for very helpful discussions: David Atwood, Bill Bardeen, Richard Blankenbecler, Eric Braaten, Stan Brodsky, Helen Perk, Jacques Perk, Tom Rizzo, Davison Soper, George Sudarshan, Levan Surguladze, Alan White, and Cosmos Zachos. This work was supported by the U.S. Department of Energy under Grant No. DE-FG02-94ER40852. 


\section{REFERENCES}

(1) M. A. Samuel, G. Li, and E. Steinfields, Phys. Rev. D48, 869 (1993).

(2) M. A. Samuel, G. Li, and E. Steinfields, "On Estimating Perturbative Coefficients in Quantum Field Theory, Condensed Matter Theory, and Statistical Physics", Oklahoma State University Research Note 278, August (1993).

(3) M. A. Samuel, G. Li, and E. Steinfields, Phys. Lett. B323, 188 (1994); M. A. Samuel and G. Li, "Estimating Perturbative Coefficients in High Energy Physics and Condensed Matter Theory", International Journal of Theoretical Physics (1994).

(4) M. A. Samuel and G. Li, Phys. Lett. B331, 114 (1994).

(5) M. A. Samuel and G. Li, "On the $R$ and $R_{\tau}$ Ratios at the Five-Loop Level of Perturbative QCD", SLAC-PUB-6370, October (1993).

(6) J. Zinn-Justin, Physics Reports 1, 55 (1971).

(7) J. Nutall, J. Math. Anal. 31, 147 (1970).

(8) G. A. Baker, Jr. Essentials of Padé Approximants, (Academic, New York, 1975).

(9) C. Bender and S. Orzag, Advanced Mathematical Methods for Scientists and Engineers, (McGraw-Hill, New York, 1978).

(10) C. Chlouber, G. Li, and M. A. Samuel, "Padé Approximants - Type I and Type II - and Their Application", Oklahoma State University Research Note 265, February (1992).

(11) M. A. Samuel, "On Estimating Perturbative Coefficients in Quantum Field Theory and Statistical Physics", Oklahoma State University Research Note 290, May (1994).

(12) G. Arfken, Mathematical Methods of Physics, (Academic, New York, 1985).

(13) M. Abramowitz and I.A. Stegun, Handbook of Mathematical Functions, (U.S. Gov't Printing Office, Wash., D.C., 1964).

(14) I. S. Gradshteyn and Ryzhik, Tables of Integrals, Series, and Products, 
(Academic, New York, 1980).

(15) S. A. Larin, T. van Ritbergen, and J. A. M. Vermaseren, "The Next-Next-toLeading QCD Approximation for Nonsinglet Moments of Deep Inelastic Structure Functions", NIKHEF-H-93-29, December (1993).

(16) C. Domb, Ising Model in Phase Transitions and Critical Phenomena, vol. 3, ed. by C. Domb and M. S. Green, (Academic, New York, 1974). 
TABLE I. Padé estimates for ${ }_{2} F_{1}$.

[N/M]

[4/4]

[5/5]

$[6 / 6]$

[7/7]

[8/8]

[9/9]

[10/10]

[11/11]

[12/12]

[13/13]

[14/14]

[15/15]

[16/16]

[17/17]

[18/18]
TESTMIN

$0.649 \times 10^{-8}$

$0.105 \times 10^{-9}$

$0.103 \times 10^{-11}$

$0.100 \times 10^{-13}$

$0.107 \times 10^{-15}$

$0.136 \times 10^{-17}$

$0.173 \times 10^{-19}$

$0.284 \times 10^{-21}$

$0.442 \times 10^{-23}$

$0.757 \times 10^{-25}$

$0.151 \times 10^{-26}$

$0.652 \times 10^{-30}$

$0.197 \times 10^{-29}$

$0.705 \times 10^{-30}$

$0.192 \times 10^{-29}$

\section{TESTMAX}

0.649

34.0

2.57

7.0

0.494

$0.687 \times 10^{-2}$

$0.332 \times 10^{-3}$

$0.151 \times 10^{-4}$

$0.215 \times 10^{-5}$

$0.294 \times 10^{-6}$

$0.386 \times 10^{-7}$

$0.492 \times 10^{-8}$

$0.611 \times 10^{-9}$

$0.720 \times 10^{-10}$

$0.847 \times 10^{-11}$ 
TABLE II. Padé estimates for ${ }_{1} F_{1}$.

[N/M]

[4/4]

[5/5]

$[6 / 6]$

[7/7]

$[8 / 8]$

[9/9]

[10/10]

[11/11]

[12/12]

[13/13]

[14/14]

[15/15]

[16/16]

[17/17]

[18/18]

[19/19]

[20/20]
TESTMIN

$0.536 \times 10^{-5}$

$0.170 \times 10^{-6}$

$0.195 \times 10^{-6}$

$0.251 \times 10^{-8}$

$0.124 \times 10^{-7}$

$0.878 \times 10^{-8}$

$0.835 \times 10^{-9}$

$0.168 \times 10^{-8}$

$0.148 \times 10^{-9}$

$0.394 \times 10^{-10}$

$0.419 \times 10^{-11}$

$0.157 \times 10^{-11}$

$0.530 \times 10^{-13}$

$0.393 \times 10^{-13}$

$0.984 \times 10^{-14}$

$0.259 \times 10^{-14}$

$0.158 \times 10^{-14}$
TESTMAX

$0.246 \times 10^{-6}$

6.721

35.4

4.56

0.456

$0.102 \times 10^{-1}$

$0.114 \times 10^{-2}$

$0.697 \times 10^{-3}$

$0.260 \times 10^{-3}$

$0.270 \times 10^{-3}$

$0.676 \times 10^{-5}$

$0.119 \times 10^{-5}$

$0.844 \times 10^{-6}$

$0.121 \times 10^{-6}$

$0.201 \times 10^{-7}$

$0.217 \times 10^{-8}$

$0.128 \times 10^{-8}$ 
TABLE III. Padé estimates for ${ }_{2} F_{0}$.

[N/M] TESTMIN

\section{TESTMAX}

[4/4]

$0.146 \times 10^{-4}$

127.3

[5/5]

$0.118 \times 10^{-4}$

45.3

$[6 / 6]$

$0.458 \times 10^{-6}$

0.304

[7/7]

$0.259 \times 10^{-5}$

3.00

[8/8]

$0.986 \times 10^{-8}$

$0.307 \times 10^{-1}$

[9/9]

$0.262 \times 10^{-8}$

$0.247 \times 10^{-1}$

[9/10]

$0.528 \times 10^{-8}$

$0.227 \times 10^{-1}$

[10/9]

$0.572 \times 10^{-9}$

$0.298 \times 10^{-2}$

[10/10]

$0.590 \times 10^{-8}$

$0.152 \times 10^{-1}$

[11/11]

$0.165 \times 10^{-8}$

$0.774 \times 10^{-1}$

[13/13]

$0.132 \times 10^{-10}$

$0.573 \times 10^{-4}$

[13/14]

$0.298 \times 10^{-10}$

$0.227 \times 10^{-3}$

[14/13]

$0.214 \times 10^{-10}$

$0.776 \times 10^{-3}$

[16/16]

$0.136 \times 10^{-11}$

$0.151 \times 10^{-5}$

[17/17]

$0.377 \times 10^{-13}$

$0.105 \times 10^{-6}$

[18/18]

$0.272 \times 10^{-13}$

$0.275 \times 10^{-7}$

[19/19]

$0.349 \times 10^{-14}$

$0.729 \times 10^{-8}$

[20/20]

$0.296 \times 10^{-16}$

$0.198 \times 10^{-8}$ 
TABLE IV. Padé estimates for $\mathrm{C}_{\mathrm{L}, 2}$.

\begin{tabular}{llll}
\hline Estimate & Error & Exact & | Estimate - Exact I \\
\hline$\underline{N_{f}=3}$ & & & \\
1046 & 1046 & 2230 & 1184 \\
82,812 & 205,498 & - & \\
$\underline{N_{f}=4}$ & & & \\
837 & 837 & 2313 & 1476 \\
82,233 & 32,915 & - & 1768 \\
$\underline{N_{f}=5}$ & & 2420 & \\
652 & 652 & - & \\
80,203 & 13,522 & & \\
\hline
\end{tabular}


TABLE V. Padé estimates for $C_{L, 4}$.

\begin{tabular}{llll}
\hline Estimate & Error & Exact & $\mid$ Estimate - Exact $\mid$ \\
\hline$\underline{\mathbf{N}_{f}=3}$ & & & \\
1376 & 668 & 1473 & 137 \\
56,946 & 29,021 & - & - \\
$\mathbf{N}_{f=4}$ & & & 60 \\
1106 & 553 & 1166 & - \\
39,468 & 19,846 & - & 16 \\
$\underline{\mathbf{N}_{f}=5}$ & & 881 & - \\
897 & 449 & - & \\
25,076 & 4004 & &
\end{tabular}


TABLE VI. Padé estimates for $\mathrm{C}_{\mathrm{h}, 6}$.

\begin{tabular}{llll} 
Estimate & Error & Exact & |Estimate - Exact| \\
\hline$\underline{N_{f}=3}$ & & & \\
2305 & 1153 & 1433 & - \\
41,989 & 17,795 & - & \\
$\mathrm{N}_{f=4}$ & 1009 & 1159 & 859 \\
2018 & 12,654 & - & - \\
27,443 & & & - \\
$\underline{N_{f}=5}$ & 875 & 905 & - \\
1750 & 8434 & - & \\
15,894 & & & \\
\hline
\end{tabular}


TABLE VII. Padé estimates for $\mathrm{C}_{\mathrm{L}, 8}$.

\begin{tabular}{|c|c|c|c|}
\hline Estimate & Error & Exact & |Estimate - Exact | \\
\hline \multicolumn{4}{|l|}{$\underline{N_{f}=3}$} \\
\hline 1437 & 719 & 1985 & 548 \\
\hline 124,711 & 78,967 & - & - \\
\hline \multicolumn{4}{|l|}{$\underline{N}_{r}=4$} \\
\hline 1226 & 613 & 2043 & 817 \\
\hline 130,095 & 139,494 & - & - \\
\hline \multicolumn{4}{|l|}{$N_{c}=5$} \\
\hline 1031 & 516 & 2118 & 1087 \\
\hline 133,699 & 814,125 & $\underline{-}$ & $=$ \\
\hline
\end{tabular}


TABLE VIII. Padé estimates for $\gamma_{2}$.

\begin{tabular}{llll}
\hline Estimate & Error & Exact & |Estimate - Exact | \\
\hline$\underline{N_{f}=3}$ & & & \\
424 & 212 & 448 & 24 \\
5159 & 2270 & - & \\
$\underline{N_{f}=4}$ & & & \\
358 & 179 & 306 & 52 \\
2607 & 636 & - & 136 \\
$\underline{N_{f}=5}$ & 149 & 162 & \\
298 & 238 & - & \\
677 & & & \\
\hline
\end{tabular}


TABLE IX. Padé estimates for $\gamma_{4}$.

\begin{tabular}{llll}
\hline Estimate & Error & Exact & | Estimate - Exact | \\
\hline$\underline{\mathrm{N}_{\mathrm{f}}=3}$ & & & \\
636 & 318 & -162 & - \\
8606 & 3146 & & \\
$\underline{\mathrm{N}_{\mathrm{f}}=4}$ & & & \\
517 & 259 & 503 & 14 \\
4953 & 387 & - & \\
$\underline{\mathrm{N}_{\mathrm{f}}=5}$ & & & \\
410 & 205 & 239 & \\
954 & 297 & - & \\
\hline
\end{tabular}


TABLE X. Padé estimates for $\gamma_{6}$.

\begin{tabular}{llll}
\hline Estimate & Error & Exact & | Estimate - Exact | \\
\hline$\underline{N_{f}=3}$ & & & \\
744 & 372 & 946 & 202 \\
10,676 & 4001 & - & \\
$\underline{N_{f}=4}$ & & & \\
596 & 298 & 621 & 25 \\
5245 & 1266 & - & 174 \\
$\underline{N_{f}=5}$ & & 290 & \\
464 & 232 & - & \\
1201 & 348 & & \\
\hline
\end{tabular}


TABLE XI. Padé estimates for $\gamma_{8}$.

\begin{tabular}{|c|c|c|c|}
\hline Estimate & Error & Exact & |Estimate - Exact | \\
\hline \multicolumn{4}{|l|}{$\underline{N_{f}=3}$} \\
\hline 833 & 417 & 1081 & 248 \\
\hline 12,225 & 4629 & - & - \\
\hline \multicolumn{4}{|l|}{$\underline{N_{f}=4}$} \\
\hline 662 & 331 & 709 & 47 \\
\hline 6018 & 2552 & - & - \\
\hline \multicolumn{4}{|l|}{$\underline{N_{f}=5}$} \\
\hline 510 & 255 & 330 & 180 \\
\hline 1401 & 393 & $\ldots$ & - \\
\hline
\end{tabular}


TABLE XII. Padé estimates for the low temperature ferromagnetic susceptibility coefficients in the Ising model (he lattice).

\begin{tabular}{llll} 
Estimate & Error & Exact & |Estimate - Exact $~$ \\
\hline 8749 & 818 & 8792 & 43 \\
35,682 & 120 & 35,622 & 60 \\
143,333 & 447 & 143,079 & 254 \\
569,470 & 950 & 570,830 & 1360 \\
$2,264,740$ & 631 & $2,264,649$ & 91 \\
$8,942,853$ & 2031 & $8,942,436$ & 417 \\
$35,159,776$ & 8724 & $35,169,616$ & 9840 \\
$137,838,225$ & 5787 & $137,839,308$ & 1083 \\
$538,596,320$ & 10,430 & & \\
\hline
\end{tabular}


TABLE XIII. Padé estimates for the low temperature ferromagnetic susceptibility coefficients in the Ising model (sq lattice).

\begin{tabular}{llll} 
Estimate & Error & Exact & $\mid$ Estimate - Exact $\mid$ \\
\hline 449 & 138 & 416 & 33 \\
2715 & 830 & 2791 & 76 \\
18,699 & 1592 & 18,296 & 403 \\
118,069 & 35,392 & 118,016 & 53 \\
751,928 & 146 & 752,008 & 80 \\
$4747 \times 10^{3}$ & $1410 \times 10^{3}$ & $4746 \times 10^{3}$ & $O\left(10^{3}\right)$ \\
$2973 \times 10^{4}$ & $721 \times 10^{4}$ & $2973 \times 10^{4}$ & $O\left(10^{3}\right)$ \\
$18,502 \times 10^{4}$ & $5494 \times 10^{4}$ & & -
\end{tabular}

